\title{
NEURAL NETWORK MODELLING OF THE MECHANICAL PROPERTIES OF NICKEL BASE SUPERALLOYS.
}

\author{
J. Jones* and D.J.C. MacKay\# \\ ${ }^{*}$ Department of Materials Science and Metallurgy, University of Cambridge, Cambridge, UK. \\ \# Department of Physics, University of Cambridge, Cambridge, UK.
}

\begin{abstract}
Modelling techniques are being developed with the aim of reducing the cost and time associated with the development of new alloys for critical aerospace components. In this paper the yield and tensile strengths of commercial polycrystalline wrought $\mathrm{Ni}$ base supcralloys are modelled using an artificial neural network technique. Neural networks of this type are capable of realising a great variely of non-linear relationships of considerable complexity. They are 'trained' using existing experimental data which is presented to the network in the form of input-output pairs, thus the optimum relationship is found between the tensile properties and those parameters which are considered to be of importance. Through a series of tests it was found that with appropriate training a neural network can reliably reproduce metallurgical experience and knowledge. These results demonstrate that neural network models could be successfully used in the development of new alloys, reducing the amount of experimental work required and thus the time taken for a new alloy to be introduced into service.
\end{abstract}

\section{Introduction}

The yield and tensile strengths of nickel-base superalloys are fundamental design parameters in the manufacture of aerospace components. Today's highly alloyed, complex superalloys derive their outstanding tensile properties from a combination of several principal strengthening mechanisms (1): solid solution (2-5), precipitation (6-8) and grain boundary $(9,10)$ strengthening. Consequently, there are a large number of variables that can influence these tensile properties. This complexity in the nickel superalloy system makes them very useful in the aerospace industry, but at the same time very difficult to design. Quantitative models can be very useful in this respect, assisting the development of new alloys by reducing the need for experimental work. Although many mechanisms are known to contribute to the

$$
\text { Superalloys } 1996
$$

strength of Ni-base superalloys it has not yet been possible to incorporate these mechanisms into a single model capable of predicting the tensile properties of complex industrial alloys. Already there is a vast experimental database for these properties, both in the published literature and in industrial data banks. This paper presents an investigation into the development of quantitative models of the tensile properties of Ni-base superalloys using an artificial neural network.

An artificial neural network is a computer intensive blind modelling technique which has the ability to recognise patterns in complex and often noisy experimental data $(11,12)$. They first emerged as self learning tools in the early 1940's inspired by the architecture and learning capacity of the brain. Considerable development has occurred since then resulting in a wide range of neural network applications such as image and speech recognition, on-line control engineering, medicine, business and finance and many others (13).

\section{Neural Network Approach}

Neural networks are parameterized non-linear models used for empirical regression and classification modelling. Their flexibility enables them to discover more complex relationships in data than traditional statistical models which often assume a linear dependence of the predicted 'output' variable on the given 'input' variables. Neural networks are able to implement more general (and more complex) non-linear relationships thereby finding the optimum relationship automatically, irrespective of the complexity with which the variables may be associated. Only the input and output data must be presented to the network, which is a major advantage compared to traditional regression analysis where a prior choice of relationship must be made.

A neural network is composed of an interconnected array of simple processing units, referred to as nodes, arranged in a pattern analogous to the network of neurons and synapses in the brain. 
The strength of the neural network lies in the large number of connections, each having an associated weight or strength which is adapted during training to improve performance. While the behaviour of a single node is relatively simple, the systematic behaviour of the nodes can be very sophisticated.

A network of the type shown in fig. 1 has been used in this analysis. It consists of $n$ input variables which are considered to control the output and two layers of nodes; a hidden layer of variable size and the output node.

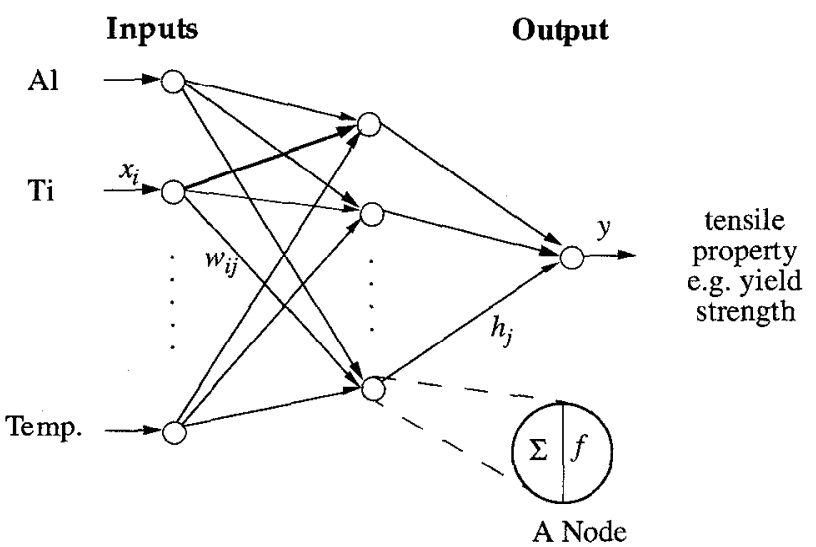

Figurc 1: Schematic diagram of a ncural network.

Each hidden node receives a contribution from every input node, $x_{i}$ modified only by the connection weights, $w_{i j}$. The contributions are summed and passed through a non-linear 'sigmoidal' transfer function giving a node output $h_{j}$ :

$$
h_{j}=\tanh \left(\sum_{i} w_{i j} x_{i}+\theta_{j}\right)
$$

where $\theta_{j}$ is a constant bias term. The non-linear nature of this function is necessary for the algorithms used during the training process (12). This sum, $h_{j}$ is then passed on to the output node. At the output layer a linear transfer is performed giving an output $y$ :

$$
y=\sum_{j} w_{j l} h_{j}+\theta_{l}
$$

As with regression analysis the neural network solution can be summarised by a series of coefficients or weights and a specification of the type of function relating the inputs to the output.

The connection weights are determined through a supervised 'training' process where real experimental data sets are presented to the network as model input - target output pairs. In response, the weights are adapted through a minimisation of a regularised sum of squared errors, $M(w)$ where:

$$
M(w)=\beta E_{t}+\alpha E_{w}
$$

where

$$
E_{t}=0.5 \sum_{n}(t-y)^{2} \text { and } E_{w}=\sum_{k} \frac{w_{k}^{2}}{2}
$$

where $t$ is the target output, $y$ is the network output, $n$ is the number of training data set, $w_{k}$ is a network weight, $k$ is the number of weights of connections coming from all the input nodes. $\alpha$ and $\beta$ are regularising constants. The objective function $M(w)$ consists of two terms $E_{t}$ and $E_{w} . E_{t}$ is a measure of the error in the network, that is the difference between the target output, $t$ and the network output, $y$ summed over all the data points in the training set. The second term $E_{w}$ is designed to reduce the detrimental effect of any input variables present which do not affect the output. Further details of this procedure are described in more detail elsewhere $(12,14,15)$. Thus the optimum non-linear interpolant that fits the data well is found.

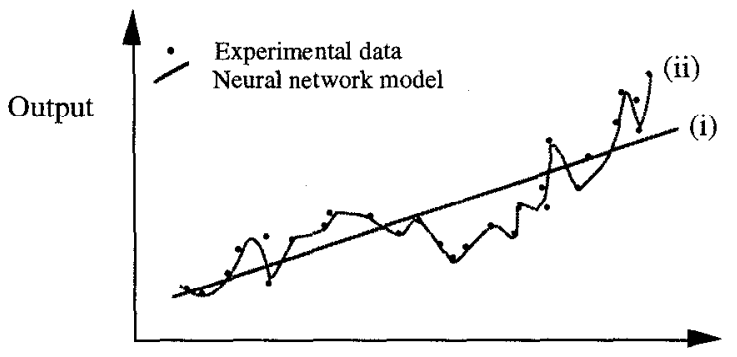

Input space

(a) (i) Insufficient number of hidden nodes,

(ii) Excessive number of hidden nodes.

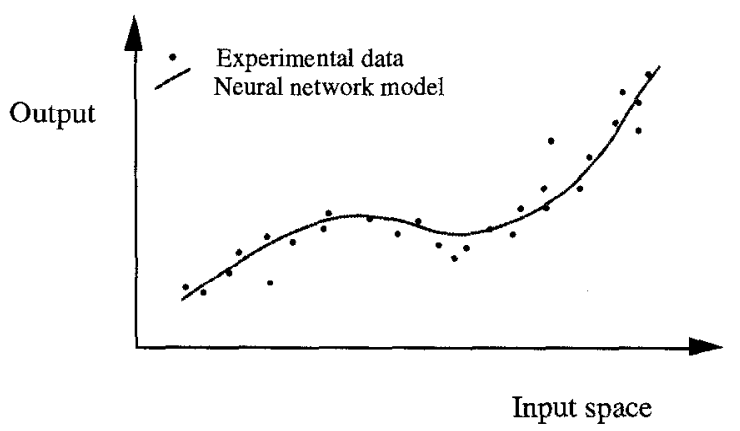

(b) Optimum network size.

Figure 2: Effect of hidden layer size on generalisation.

The integrity of a trained network lies in its ability to generalise, that is its performance on the actual problem once training is complete. Blind modelling of this type can be susceptible to over representation in which even interpolation becomes dangerous. In effect, the network learns the data used in the training process but not the general principles controlling the output. Obviously the complexity of the model is dependent on the 
number of hidden layer nodes and the regularisation constants $\alpha$ and $\beta$. The effect of hidden layer size on generalisation is shown schematically in fig.2. If too few nodes are used, fig.2(a), then the network may not train or will form a very simplistic model which does not represent the data well. Conversely, if too many nodes are used, fig.2(a), specific examples in the training set will be learnt and the resulting network will not generalise. Therefore an optimal number of nodes must be selected, fig.2(b).

Traditionally the danger of over representation is reduced by dividing a given data base into two representative sets. The network is then trained on one set and subsequently tested on the other to assess the networks generalisation. In addition, MacKay $(14,15)$ has developed a neural network method which incorporates a Bayesian statistical framework allowing objective choices of the network parameters such as hidden layer size and regularisation constants. Therefore, the model complexity can be controlled thus enabling a network to be trained and used more efficiently and successfully. Using this method it is also possible to automatically identify which of the many possible input parameters are in fact important in the regression.

Once the network has been fully trained, estimation of the output for any given set of inputs is very rapid. These predictions are accompanied by error bars which depend on the specific position in input space, quantifying the model's certainty about its predictions. This is a further advantage over traditional regression analysis where a single global error bar is calculated.

\section{Neural Network Modelling of Mechanical Properties}

A large computer data base was assembled for the yield strength and ultimate tensile strength (UTS) for a range of commercial, wrought, polycrystalline alloys tested over a range of temperatures (16). The input parameters were the alloy chemistry (e.g. wt.\% Al, $\mathrm{Ti}, \mathrm{Cr}$ etc.) and temperature. Table I shows the range of the 16 input variables, the yield strength and UTS.

Two networks were employed, with 16 input variables each corresponding to one of those listed in table I, one giving an output of yield strength and the other UTS. For comparison purposes, each of the 16 inputs and the output was scaled linearly between +0.5 and -0.5 , this prevents domination by a few large input variables. This normalisation process can be expressed quantitatively as:

$$
x_{n}=\frac{x-x_{\min }}{x_{\max }-x_{\min }}-0.5
$$

where $x_{n}$ is the normalised value of $x, x_{\min }$ and $x_{\max }$ are the minimum and maximum values of $x$ in the entire data set as listed in table I.

The database consisted of $\sim 200$ points of which 100 were used to train the network and the remaining data points reserved to test the trained network. The training and test data were randomly taken from the master database. The size of the hidden layer was varied between 2 and 8 hidden nodes. Every network size was trained several times, each with a different set of initial random weights.

The performance of each network was quantified by calculating the root mean squared (RMS) error in the predicted output for both the training and test set. The RMS error is given by:

$$
E=\sqrt{\frac{\sum[y-t]^{2}}{n}}
$$

where $n$ is the number of data points, $t$ is the target experimental output and $y$ is the output of the trained network.

Table I: Range of input and output data included in the data base. All compositions are given in wt.\%.

\begin{tabular}{|c|c|c|c|c|c|c|c|}
\hline Input & Minimum & Maximum & Mean & Input & Minimum & Maximum & Mean \\
\hline $\mathrm{Ni}$ & 38 & 76 & 57 & $\mathrm{Mn}$ & 0 & 0.5 & 0.16 \\
\hline $\mathrm{Cr}$ & 12 & 30 & 18.7 & $\mathrm{Si}$ & 0 & 0.5 & 0.13 \\
\hline $\mathrm{Co}$ & 0 & 20 & 8.4 & $\mathrm{C}$ & 0.03 & 0.35 & 0.08 \\
\hline $\mathrm{Mo}$ & 0 & 10 & 4.2 & $\mathrm{~B}$ & 0 & 0.16 & 0.01 \\
\hline $\mathrm{W}$ & 0 & 6.0 & 0.5 & $\mathrm{Zr}$ & 0 & 0.2 & 0.02 \\
\hline $\mathrm{Ta}$ & 0 & 1.5 & 0.05 & Temp. $\left({ }^{\circ} \mathrm{C}\right)$ & 21 & 1093 & 614 \\
\hline $\mathrm{Nb}$ & 0 & 6.5 & 0.43 & & & & \\
\hline $\mathrm{Al}$ & 0 & 4.9 & 1.7 & Output (MPa) & & & \\
\hline $\mathrm{Ti}$ & 0 & 5.0 & 2.1 & Yield strength & 28 & 1310 & - \\
\hline $\mathrm{Fe}$ & 0 & 40 & 7.1 & UTS & 35 & 1620 & - \\
\hline
\end{tabular}




\section{Results and Discussion}

As expected the performance on the training set for both networks is improved with hidden layer size, figs.3\&4. The network outputs approach the target experimental results as the complexity of the network increases. However, more importantly the RMS error in the test set also decreases with increasing network size reaching an asymptotic limit at about 6 hidden nodes in both models, beyond which the model does not improve with increasing complexity. Therefore the optimum solution, with best generalisation, was selected as models with 6 hidden nodes. The yield and tensile strength data of these alloys have been modelled within an error of 60 and $70 \mathrm{MPa}$ respectively. The level of agreement achieved in both the training and test set is shown in figs. $5 \& 6$, each predicted value is accompanied by $a \pm 1 \sigma$ error bar. The magnitude of the error bar is dependent on the position in input

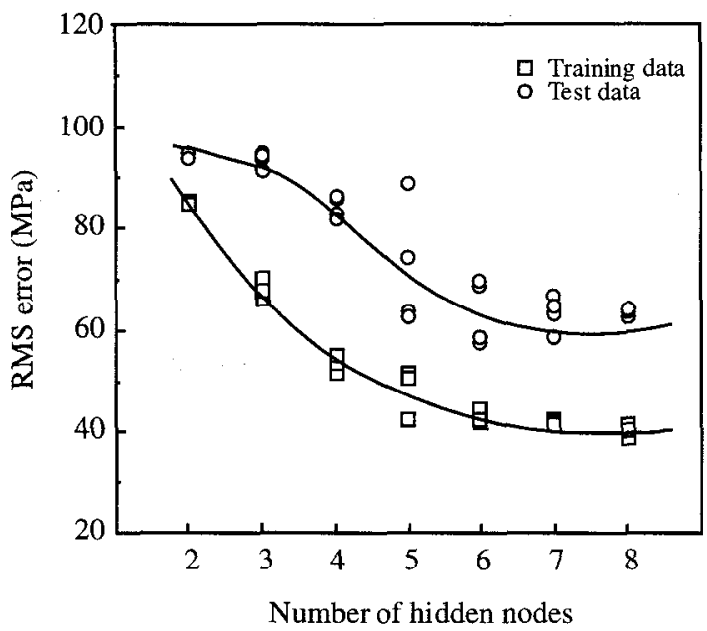

Figure 3: Performance on the yield strength training and test set as a function of the number of hidden nodes.

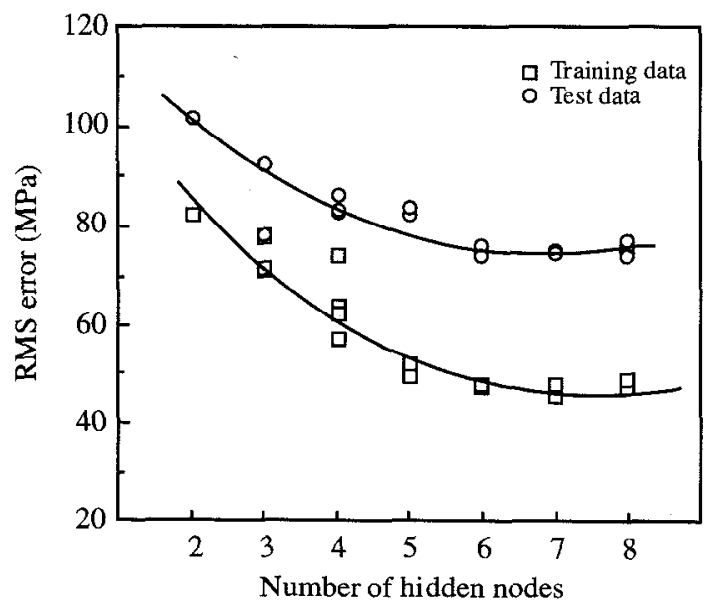

Figure 4: Performance on the UTS training and test set as a function of the number of hidden nodes. space and is a measure of how well the model is defined for that given set of input variables. A proportion of the noise in the models is believed to be real noise, that is, deviations in the tensile data which are not fully explained by the input parameters. It is believed that this could be reduced by inclusion of additional microstructural input parameters such as the $\gamma$ volume fraction, size and grain size (17).

The trained models were then subjected to a variety of metallurgical tests to check they had learnt the correct science rather than nonsensical trends. Fig.7 illustrates the model perceived "significance" of each input variable in both datasets. This can be considered as a partial correlation coefficient, that is it indicates the fraction of variation explained by each input variable.

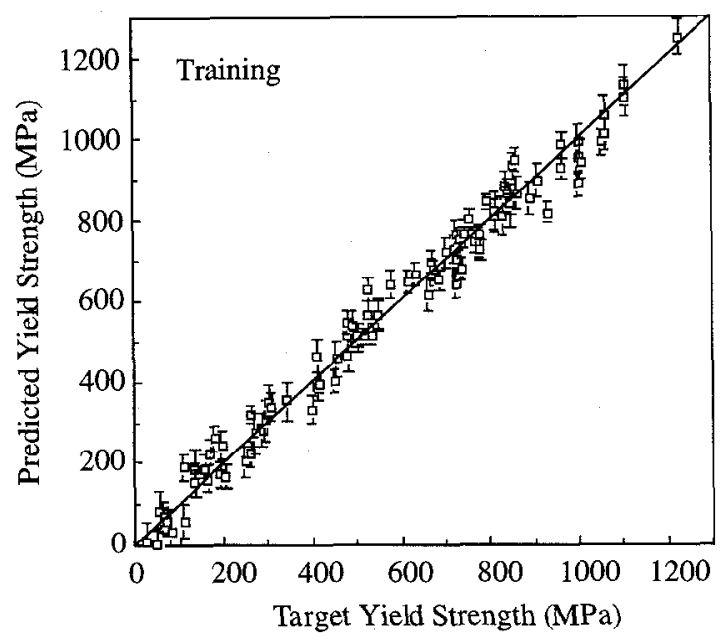

(a): Training set.

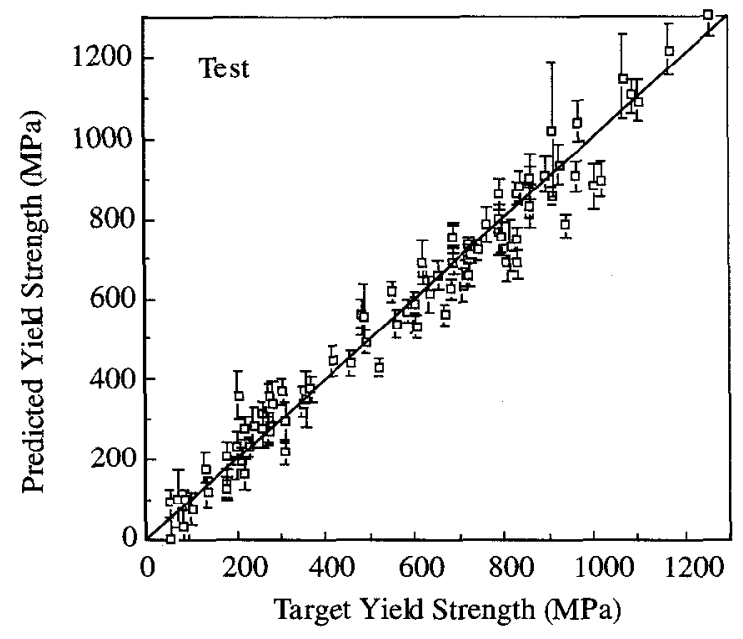

(b): Test set.

Figure 5: Pcrformance of the optimum yield strength model on the training and test set with $\pm 1 \sigma$ error bars. 


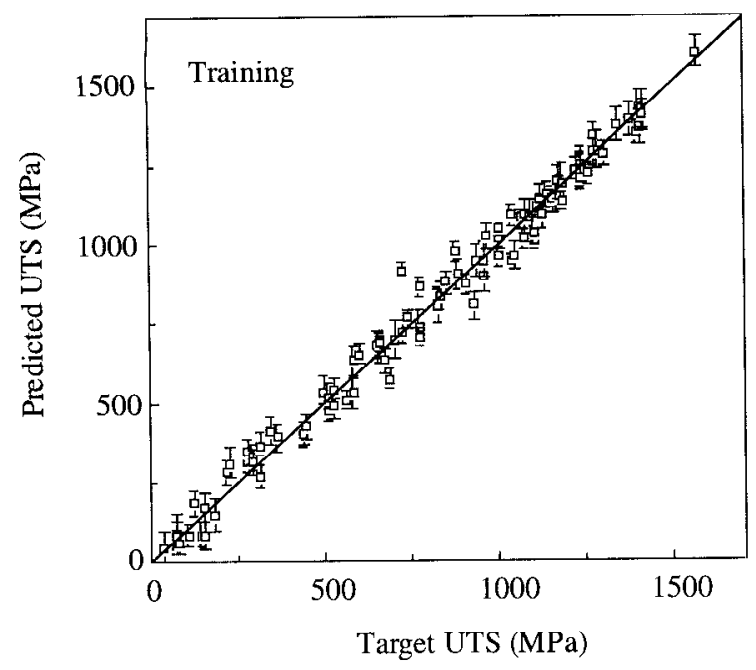

(a): Training set.

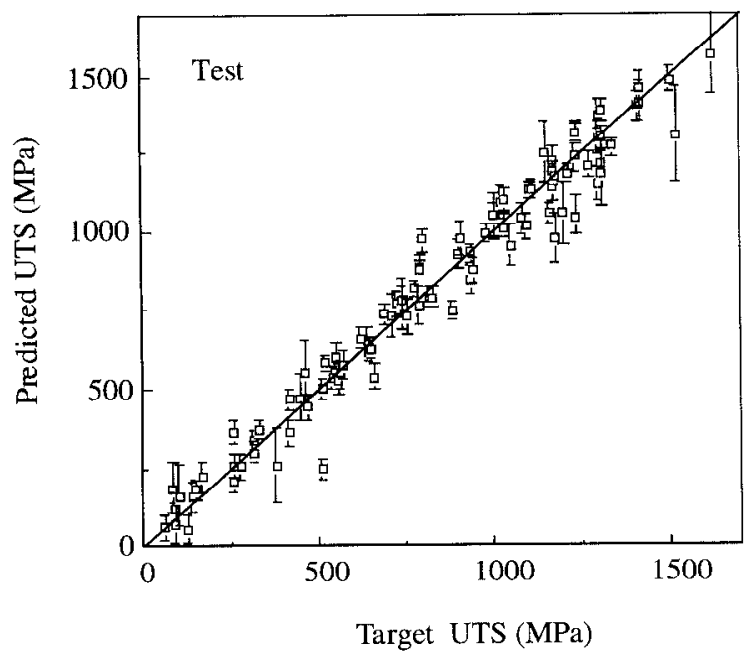

Not surprisingly temperature is found to be the most significant input variable; the variation in temperature from 25 $1100^{\circ} \mathrm{C}$ in the dataset is expected to lead to significant changes in microstructure as at elevated temperatures $\left(>600^{\circ} \mathrm{C}\right)$ superalloys are chemically dynamic systems, the phases constantly reacting and interacting (18). The significant alloying elements identified by the network successfully reflect the active strengthening mechanisms in the superalloy system. Precipitation strengthening of the $\gamma$ matrix by $\gamma^{\prime}$ is the dominant strengthening mechanism in the majority of superalloys and hence the predominant $\gamma^{\prime}$ formers $\mathrm{Ti}$, $\mathrm{Al}$ and $\mathrm{Nb}$ are found to be the most significant alloying elements. Significant contributions are also made by Mo and $\mathrm{W}$ the solid solution strengthening elements, at elevated temperatures $(>0.6 \mathrm{Tm}$, where $T \mathrm{~m}$ is the absolute melting temperature) $\gamma$ strengthening is also diffusion dependent therefore Mo and $\mathrm{W}$ are also beneficial due to their low diffusivity in the nickel rich matrix. Minor effects of $\mathrm{C}, \mathrm{B}$ and $\mathrm{Fe}$ are also detected.

The physical significance of the models was further tested by performing a series of predictions to investigate the sensitivity of yield strength and UTS to compositional and temperature variations. A selection of 'experiments' are shown in figs.8(a-d). All predictions are accompanied with $\pm 1 \sigma$ error bars. These predictions are similar to the matrix of experimental alloys prepared and tested during the design of a new alloy.

The temperature dependence of the tensile properties of superalloys is correctly predicted as shown in fig.8(a). The softening of the $\gamma$ matrix is offset by the remarkable reversible increase in strength of $\gamma$ with increasing temperature $(19,20)$.

(b): Test set.

Figure 6: Performance of the optimum UTS model on the training and test set with $\pm 1 \sigma$ error bars.

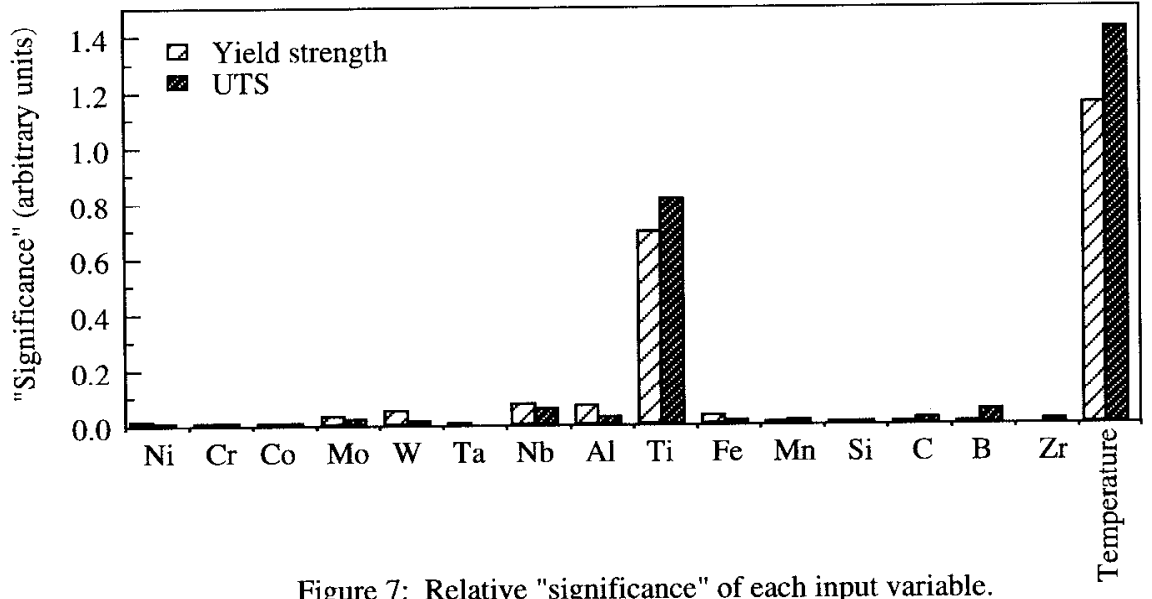

Figure 7: Relative "significance" of each input variable. 


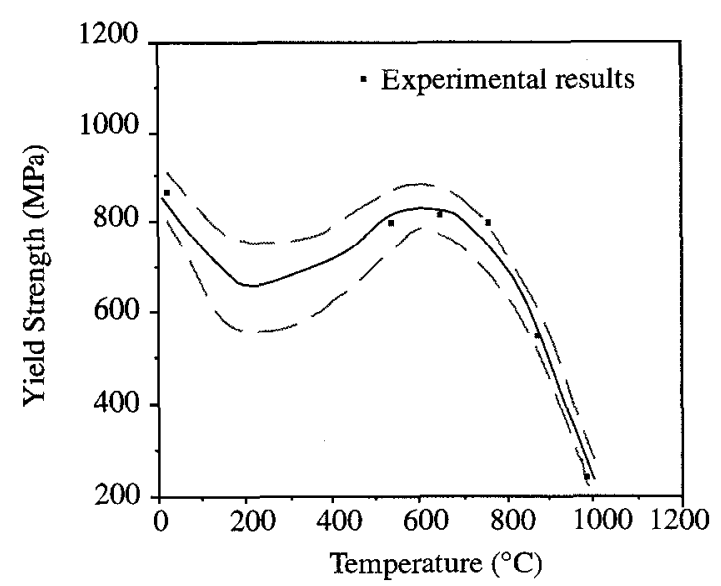

Figure 8(a): Predicted temperature dependence of the yield strength of a $\gamma / \gamma$ superalloy.

It was perhaps surprising that $\mathrm{Ti}$ received such a high significance value compared to Al the other major $\gamma^{\prime}$ forming element, fig.7. The relative strengthening effect of $\mathrm{Ti}$ and $\mathrm{Al}$ was investigated by systematically altering the ratio of $\mathrm{Al}$ to ' $\mathrm{T}$ ' $\mathrm{I}$ atoms in a superalloy. In the test series the total $\mathrm{Al}+\mathrm{Ti}$ content was kept constant, thus the total fraction of $\gamma$ ' is approximately unaltered. An increase in $\mathrm{Ti} / \mathrm{Al}$ ratio is predicted to increase both the yield and tensile strength as shown in fig.8(b). This predicted trend is in agreement with experimental results of Miller and Ansell (21) on a Ni-15Cr-Mo alloy. Substitution of Ti atoms for Al atoms in the $\gamma$ lattice increases both the $\gamma$ lattice parameter, thereby increasing the lattice misfit (22) and the anti-phase boundary energy (23) of $\gamma^{\prime}$, both of these effects result in an increased $\gamma$ strengthening contribution as described by coherency and order hardening theories.

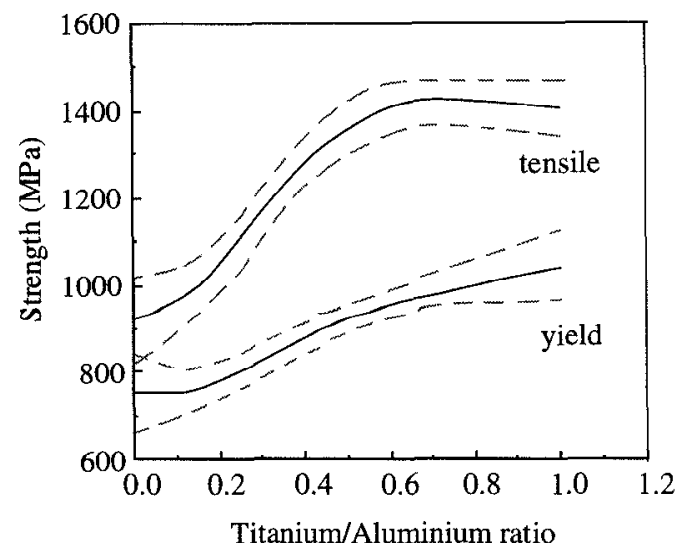

Figure 8(b): Predicted sensitivity of room temperature yield and tensile strength to variations in $\mathrm{Ti} / \mathrm{Al}$ ratio.
Many researchers have investigated experimentally the role of $\mathrm{Co}$ in superalloys for both economic and strategic reasons. The effect of reducing Co levels in Waspaloy to zero was predicted as shown in fig.8(c), comparable experimental results of Maurer et al. (24) are also given. It should be noted that the data of Maurer et al. was not included in the training or test data bases, therefore the predictions made are a good test of the model's generalisation ability. It can be seen that Co levels have very little effect on the tensile properties of Waspaloy in good agreement with the experimental results. Any small changes in yield strength have been attributed to changes in fine $\gamma^{\prime}$ volume fraction and stacking fault energies $(24,25)$.

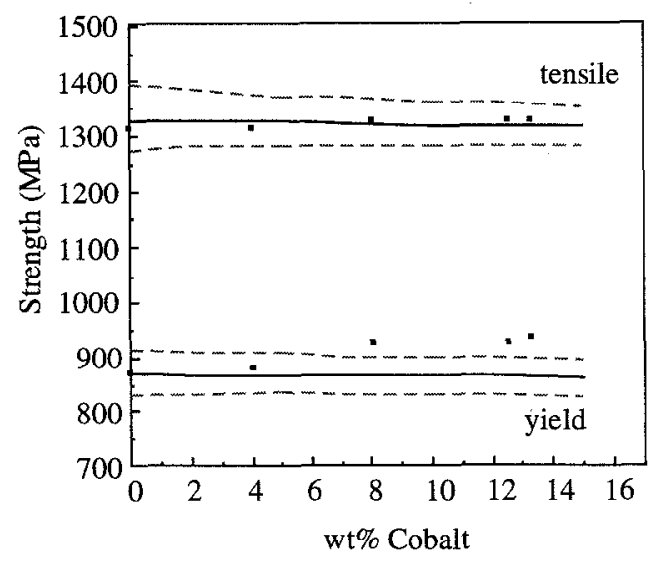

Figure 8(c): Predicted sensitivity of room temperature yield and tensile strength to variations in Co levels.

The predicted effect of Mo on the room temperature tensile properties of a typical Ni-base superalloy, namely Astroloy is shown in fig.8(d). It is evident that additions of Mo increase both the yield and tensile strength of the alloy, a $5 \mathrm{wt} . \%$ increase in the Mo content leads to a predicted increase of $\sim 200 \mathrm{MPa}$ in yield and tensile strength. There are several well documented theories to account for the strengthening effect of Mo relating to the atomic diameter oversize $(3,4)$ and elastic modulus change $(5)$ when an alloying element is introduced into the matrix and it's position in the periodic table (26), all of which have the net effect of impeding dislocation motion thereby increasing the strength. Deckcr (2) estimated the potency of solid solution elements present in the $\gamma$ matrix and concluded that Mo contributes strongly, as predicted.

Fig.8(d) also illustrates the useful nature of the quantitative error bars, they serve as a measure of how well the model is defined for a particular set of inputs. In this example it is clear that the predictions are less useful at the higher Mo contents, due to the addition of Mo well outside the range of the training data (tablc I). 


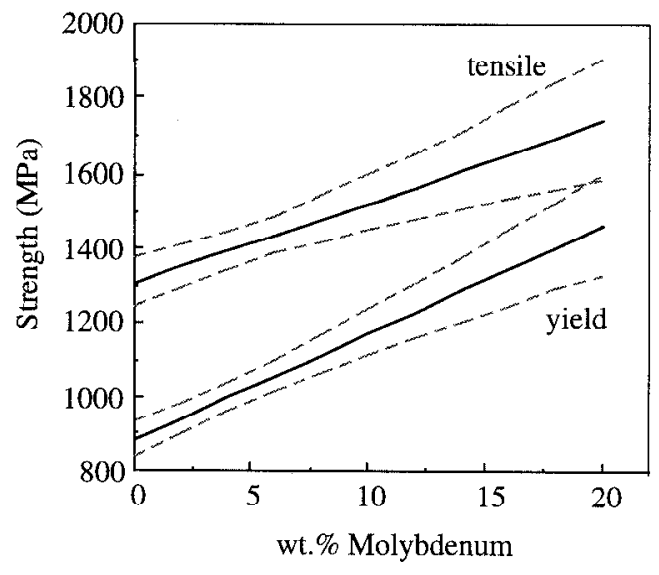

Figure $8(d)$ : Predicted sensitivity of room temperature yield and tensile strength to additions of Mo.

\section{Conclusions}

An artificial ncural network modelling technique has been successfully applied to Ni-base superalloy tensile properties. A quantitative model has been produced which can predict the yicld and tensile strengths within error bounds. Despite the empirical nature of the modelling process the resultant models are found to be consistent with current metallurgical theory and experience. These results reveal the potential of these models as predictive alloy design tools, reducing the cost and time associated with experimental work.

\section{Acknowledgements}

The authors wish to acknowledge the financial support of RollsRoyce plc. and the Engineering and Physical Sciences Research Council. They would also like to thank Professor C.J. Humphreys for the provision of research facilitics. Thanks are also due to Dr. R.C. Thomson, Dr. H.K.D.H. Bhadeshia and Dr. D.M. Knowles for useful discussion and advice.

\section{References}

1. E.W. Ross \& C.T. Sims, "Nickel Base Alloys", Superalloys II, eds. C.T. Sims et al., (New York, John Wiley \& Sons, 1987), 97-133.

2. R.F. Decker, "Strengthening Mechanisms in Nickel Base Superalloys", Climax Molybdenum Company Symposium, Zurich, May 5-6 1969, 147-170.

3. R.M.N. Pelloux \& N.J. Grant, "Solid Solution and Second Phase Strengthening of Nickel Alloys at High and Low Temperatures", Trans. AIME, 218, (1960), 232-237.
4. E.R. Parker \& T.H. Hazlett, "Principles of Solution Hardening", Relation of Properties to Microstructure, American Society of Metals, 30, (1954), 30-70.

5. R.L. Fleischer, "Substitutional Solution Hardening" Acta Metall., 11, (1963), 203-209.

6. A.J. Ardell, "Precipitation Hardening" Met. Trans., 16A, (1985), 2131-2165.

7. E. Nembach \& G. Neite, "Precipitation Hardening of Superalloys by Ordered $\gamma$-Particles", Prog. in Mat. Sci., 29, (1985), 177-319.

8. B. Reppich, "Some New Aspects Concerning Particle Hardening Mechanisms in $\gamma$ Precipitating Ni-Base Alloys I Theoretical Concept", Acta. Metall., 30, (1982), 87-94.

9. A. Lasalmonie \& J.L. Strudel, "Influence of Grain Size on the Mechanical Behaviour of Some High Strength Materials", J. of Mat. Sci., 21, (1986), 1837-1852.

10. W. Mangen \& E. Nembach, "The Effect of Grain Size on the Yield Strength of the $\gamma$ Hardened Superalloy Nimonic PE16", Acta. Metall., 37, (1989), 1451-1463.

11. R.P. Lippmann, “ An Introduction to Computing with Neural Nets", IEEE Acoustics. Speech and Signal Processing Magazine, 4, (2), (1987), 4-22.

12. D.R. Hush \& B.G. Horne, "Progress in Supervised Neural Networks", IEEE Signal Processing Magazine, 1993 (January), 8-39.

13. N. Ryman-Tubb, "Implementation, the Only Sensible Route to Wealth Creating Success: a Range of Applications", Neural Networks, Neuro-Fuzzy and Other Learning Systems for Engineering Applications and Research, ed. J.A. Powell, (London, DRAL, 1994), 37-44.

14. D.J.C. MacKay, "Bayesian Interpolation", Neural Computation, 4, (1992) 415-447.

15. D.J.C. MacKay, "A Practical Bayesian Framework for Backpropagation Networks", Neural Computation, 4, (1992), 448-472.

16. "High Temperature High-Strength Nickel Base Alloys", Distributed by Nickel Development Institute, Birmingham, England.

17. J. Jones, "Neural Network Modelling of the Tensile Properties of Ni-Base Superalloys", (CPGS dissertation, University of Cambridge, 1994).

18. P.W. Keefe, S.O. Mancuso \& G.E. Maurer, "Effects of Heat Treatment and Chemistry on the Long Term Phase Stability of a High Strength Nickel-Based Superalloy", Superalloys 1992, ed. S.D. Antolovich et al ., (The Metallurgical Society, 1992), 487-496.

19. P. Beardmore, R.G. Davies \& T.L. Johnston, "On the Temperature Dependence of the Flow Stress of Nickel-Base Alloys", Trans. AIME, 245, (1969), 1537-1545.

20. R.G. Davies \& N.S. Stoloff, "On the Yield Stress of Aged Ni-Al Alloys", Trans. AIME, 233, (1965), 714-719.

21. R.F. Miller \& G.S. Ansell, "Low Temperature Mechanical Behaviour of Ni-15Cr-Al-Ti-Mo Alloys", Met. Trans. A. 8, (1977), 1979-1991. 
22. A.K. Jena \& M.C. Chaturvedi, "The Role of Alloying Elements in the Design of Nickel-Base Superalloys", J. of Mat. Sci., 19, (1984), 3121-3139.

23. D. Raynor \& J.M. Silcock, "Strengthening Mechanisms in $\gamma$ ' Precipitating Alloys", Met. Sci. Journal, 4, (1970), 121-129.

24. G.E. Maurer, L.A. Jackman \& J.A. Domingue, "Role of Cobalt in Waspaloy", Superalloys 1980, ed. J.K. Tien, (American Society for Metals, 1980), 43-52.

25. R.N. Jarrett \& J.K. Tien, "Effects of Cobalt on Structure, Microchemistry and Properties of a Wrought Nickel-Base Supcralloy", Mct. Trans. A, 13A, (1982), 1021-1032.

26. B.E.P. Beeston \& L.K. France, "The Stacking Fault Energy of Some Binary Nickel Alloys Fundanental to the Nimonic Series", J. Inst. of Metals, 96, (1968), 105-107. 